Editor's Note: These short reviews of a recent paper in the Journal, written exclusively by graduate students or postdoctoral fellows, are intended to mimic the journal clubs that exist in your own departments or institutions. For more information on the format and purpose of the Journal Club, please see http://www.jneurosci.org/misc/ifa_features.shtml.

\title{
Adult Neural Precursor Cells and the Dysmyelinated Spinal Cord
}

\author{
Daniel J. Webber \\ University of Cambridge, Cambridge CB2 2PY, United Kingdom \\ Review of Eftekharpour et al. (http://www.jneurosci.org/cgi/content/full/27/13/3416)
}

The myelin surrounding axons is highly organized, with ion channels and adaptor molecules densely clustered at nodes of Ranvier. Loss of myelin decreases impulse conduction and ultimately leads to degeneration of axons. Demyelinated axons also have disrupted organization, with loss of $\mathrm{K}^{+}$channels contributing to functional deficits (Karimi-Abdolrezaee et al., 2004). Cell-based treatments are one approach to repair the loss of myelin and damaged axons (Ben-Hur et al., 2003; Pluchino et al., 2003; Keirstead et al., 2005).

A recent study in The Journal of Neuroscience (Eftekharpour et al., 2007) examined the role of adult neural precursor cells (aNPCs) in myelin repair (Fig. 1). As a model system, the authors used a congenitally myelin-deficient transgenic Shiverer (shi/shi) mouse with sparse myelin sheaths and altered nodal $\mathrm{K}^{+}$channels. The adult NPCs had the ability to commit to the three main CNS cell types in ratios similar to that seen by previous studies. This demonstrated potential is routine and well established for adult NPCs but nonetheless important [Eftekharpour et al. (2007), their Fig. 1 (http://www.jneurosci.org/cgi/content/ full/27/13/3416/F1)]. The authors assumed that adult NPCs were multipotent; however, clonal sphere formation was not performed. Using light and electron mi-

Received May 9, 2007; revised May 16, 2007; accepted May 16, 2007. Correspondence should be addressed to Daniel J. Webber at the above address.E-mail:djw74@cam.ac.uk.

DOI:10.1523/JNEUROSCI.2125-07.2007

Copyright $\odot$ 2007 Society for Neuroscience $\quad$ 0270-6474/07/276605-02\$15.00/0 croscopy, the authors illustrate that shi/shi mice lacked myelin basic protein (MBP) and compact myelin [Eftekharpour et al. (2007), their supplemental Fig. 1 (http:// www.jneurosci.org/cgi/content/full/27/ 13/3416/DC1)]. The transplantation was accompanied by a mitogen infusion including bFGF, EGF, and PDGF-AA, which improves the survival of transplanted cells and encourages development of oligodendrocytes (Karimi-Abdolrezaee et al., 2006). Adult NPCs survived, as represented by yellow fluorescent protein (YFP) staining, predominantly in the white matter, after transplantation into the young adult spinal cord or neonatal brain [Eftekharpour et al. (2007), their Fig. 2 (http://www.jneurosci.org/cgi/ content/full/27/13/3416/F2)]. One drawback of this transgenic mouse is the short life span ( 15 weeks) of the animal, making it difficult to assess long-term survival of the transplanted aNPCs. Using phenotypic markers and confocal analysis, the authors predominantly observed oligodendrocyte cell commitment (78 $\pm 9 \%$ ) [Eftekharpour et al. (2007), their Fig. 3 (http://www.jneurosci.org/cgi/content/ full/27/13/3416/F3)]. Only $9 \pm 3 \%$ became GFAP-positive astrocytes, which is lower than previous transplantation studies. Although not discussed by the authors, the $7 \mathrm{~d}$ mitogen infusion might explain the low percentage of GFAP+ cells. There was no evidence of neuronal or Schwann cell differentiation after transplantation of aNPCs [Eftekharpour et al. (2007), their Fig. 4 (http://www.jneurosci.
org/cgi/content/full/27/13/3416/F4)], nor was there any evidence of proliferation observed with bromodeoxyuridine labeling [Eftekharpour et al. (2007), their supplemental Fig. 3 (http://www.jneurosci. org/cgi/content/full/27/13/3416/DC1)]. The authors observed YFP+ cells that colocalized with MBP and wrapped endogenous axons [Eftekharpour et al. (2007), their Fig. 5 (http://www.jneurosci.org/ cgi/content/full/27/13/3416/F5)]. It would be interesting to know what percentage of transplanted cells expressed MBP and are wrapping axons, the distribution of transplanted cells, and whether astrocytes were found nearer the injection site. These issues could begin to address how many myelinating cells are required for functional recovery. Electron microscopy did reveal that transplanted cells that myelinate endogenous axons form nodelike structures [Eftekharpour et al. (2007), their Fig. 6 (http://www.jneurosci.org/ cgi/content/full/27/13/3416/F6)].

The novelty of the study is in the examination of the $\mathrm{K}^{+}$subunit and contactinassociated protein (Caspr) organization. In control shi/shi mice, $\mathrm{K}^{+}$subunits were absent at nodal locations. However, after transplantation, Kv1.2 subunits and contactin-associated protein showed some reorganization [Eftekharpour et al. (2007), their Fig. 7 (http://www.jneurosci. org/cgi/content/full/27/13/3416/F7)]. Quantification of the immunostained samples showed that there was no alteration in axon number, but there was more organization of Kv1.2 subunits. Further- 


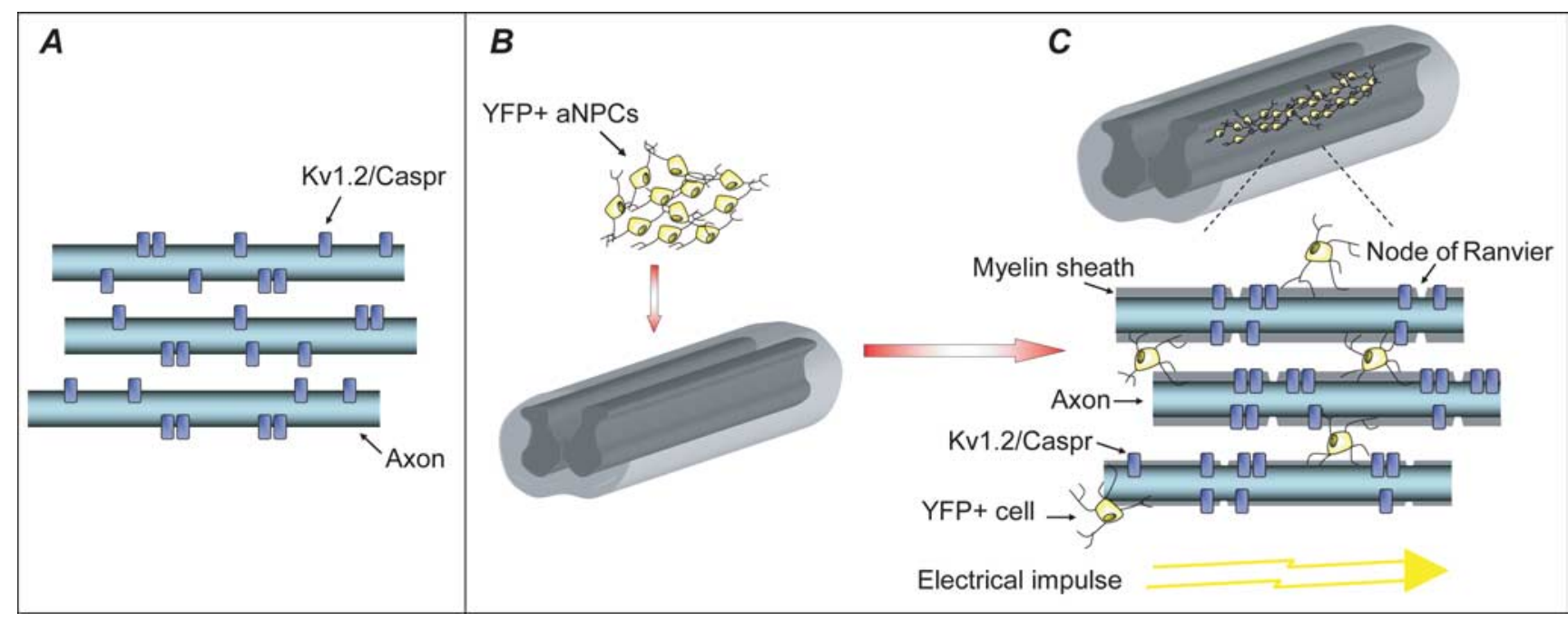

Figure 1. Myelination of congenitally dysmyelinated spinal cord axons by adult NPCs results in formation of nodes of Ranvier and improved axonal conduction. $A$, The congenitally myelindeficient transgenic shi/shi mouse lacks myelin sheaths and has altered $\mathrm{K}^{+}$channel distribution along the axons. $\boldsymbol{B}$, Eftekharpour et al. (2007) transplanted YFP + aNPCs into the spinal cord of adult shi/shi transgenic mice. Subsequent analysis 6 weeks later revealed that aNPCs survived and integrated with extensive white matter migration. Transplanted cells colocalized with oligodendrocyte and myelin markers including adenomatous polyposis coli and MBP. C, Furthermore, myelin formation in transgenic animals was accompanied by node of Ranvier formation, localization of Kv1.2 and Caspr, and improved conduction along segments of transplanted spinal cord.

more, distribution of the $\mathrm{K}^{+}$subunits over a length of axon was reduced [Eftekharpour et al. (2007), their Fig. 8 (http://www.jneurosci.org/cgi/content/ full/27/13/3416/F8)], suggesting the Kv1.2 subunits were more closely distributed and less dispersed, confirming the higher density observed at nodes in the immunomicrographs. These data were accompanied by an increase in nodal structures in shi/shi mice after transplantation of adult NPCs. Finally, the authors used electrophysiology to examine functional remyelination, something other studies often fail to show. The authors used a simple one-point recording technique with stimulating and recording electrodes only $5 \mathrm{~mm}$ apart, which showed increased amplitude, reduced latencies, and enhanced conduction velocity for segments that contained transplanted cells [Eftekharpour et al. (2007), their Fig. 9 (http://www.jneurosci.org/ cgi/content/full/27/13/3416/F9)].

It is not clear whether these observations in shi/shi mice apply to human myelin disorders. The authors argue that shi/ shi mice are more clinically relevant than other transgenic myelin-deficient models (jimpy and $m d$ ), because shi/shi have healthy oligodendrocytes that fail to produce MBP and thus myelin sheaths. It would be of interest to use a model more representative of multiple sclerosis, in which areas of normal myelin and oligo- dendrocytes are surrounded by damaged areas. For example, in experimental autoimmune encephalomyelitis (EAE), focal lesions could affect the distribution and migration of transplanted cells and molecular reconstruction. The authors discuss some of the limitations of results. For example, only $30 \%$ of the neonatal cords contained sufficient YFP + adult NPCs for quantification, suggesting that a large number of cells were lost during transplantation. Furthermore, the authors recognize that if they had given immune suppression, the survival of transplanted cells may have improved. As the authors also note, conduction velocity was comparable with md rats and EAE mice, but a twopoint recording method would be a more accurate measure.

Overall, this study demonstrates myelination, reorganization of Kv1.2 subunits and Caspr, formation of nodes of Ranvier, and improved axon conduction after transplantation of adult NPCs into the congenitally dysmyelinated cords of shi/shi transgenic mice. These results provide a platform for additional studies into alternative animal models of multiple sclerosis and the mechanisms that underlie the therapeutic benefits of stem cell transplants.

\section{References}

Ben-Hur T, Einstein O, Mizrachi-Kol R, BenMenachem O, Reinhartz E, Karussis D,
Abramsky O (2003) Transplanted multipotential neural precursor cells migrate into the inflamed white matter in response to experimental autoimmune encephalomyelitis. Glia 41:73-80.

Eftekharpour E, Karimi-Abdolrezaee S, Wang J, El Beheiry H, Morshead C, Fehlings MG (2007) Myelination of congenitally dysmyelinated spinal cord axons by adult neural precursor cells results in formation of nodes of Ranvier and improved axonal conduction. J Neurosci 27:3416-3428.

Karimi-Abdolrezaee S, Eftekharpour E, Fehlings MG (2004) Temporal and spatial patterns of Kv1.1 and Kv1.2 protein and gene expression in spinal cord white matter after acute and chronic spinal cord injury in rats: implications for axonal pathophysiology after neurotrauma. Eur J Neurosci 19:577-589.

Karimi-Abdolrezaee S, Eftekharpour E, Wang J, Morshead CM, Fehlings MG (2006) Delayed transplantation of adult neural precursor cells promotes remyelination and functional neurological recovery after spinal cord injury. J Neurosci 26:3377-3389.

Keirstead HS, Nistor G, Bernal G, Totoiu M, Cloutier F, Sharp K, Steward O (2005) Human embryonic stem cell-derived oligodendrocyte progenitor cell transplants remyelinate and restore locomotion after spinal cord injury. J Neurosci 25:4694-4705.

Pluchino S, Quattrini A, Brambilla E, Gritti A, Salani G, Dina G, Galli R, Del CU, Amadio S, Bergami A, Furlan R, Comi G, Vescovi AL, Martino G (2003) Injection of adult neurospheres induces recovery in a chronic model of multiple sclerosis. Nature 422: 688-694. 